\title{
Erratum to: Clinical evaluation of a nutraceutical diet as an adjuvant to pharmacological treatment in dogs affected by Keratoconjunctivitis sicca
}

Simona Destefanis ${ }^{1 \dagger}$, Daniela Giretto ${ }^{2 \dagger}$, Maria Cristina Muscolo ${ }^{3 \dagger}$, Alessandro Di Cerbo ${ }^{4 \dagger}$, Gianandrea Guidetti ${ }^{5}$, Sergio Canello ${ }^{5}$, Angela Giovazzino ${ }^{6}$, Sara Centenaro ${ }^{5^{*}}$ and Giuseppe Terrazzano $0^{6,7}$

\section{Erratum}

n.b. The error described below was mistakenly carried forward by the production team handling this article, and thus was not the fault of the authors.

The original version of this article [1] contained errors in Fig. 2 whereby Fig. 2b, c, d and e were missing the asterisks describing the $\mathrm{P}$ value relating to the difference in score between the 'Control' and 'Treatment' groups at the 'Post-treatment' stage.

The article has now been updated to incorporate the correct version of Fig. 2, which now displays these asterisks for each graph where appropriate.

The updated Fig. 2 can be seen below for reference.

\footnotetext{
Author details

${ }^{1}$ Clinica Veterinaria Porta Venezia, via Lambro 12, 20121 Milan, Italy. ${ }^{2}$ Clinica Veterinaria Cartesio, viale Olanda 3B, Melzo, 20066 Milan, Italy. ${ }^{3}$ Ambulatorio Veterinario Canonica, via Canonica 36, 20154 Milan, Italy. ${ }^{4}$ School of Specialization in Clinical Biochemistry, "G. d'Annunzio" University, Chieti, Italy. ${ }^{5}$ Research and Development Department, SANYpet S.p.a., Bagnoli di Sopra, Padua, Italy. ${ }^{6}$ Department of Science, University of Basilicata, Via Sauro, 85, 85100 Potenza, Italy. ${ }^{7}$ Department of Translational Medical Sciences, University of Naples Federico II, Via Pansini, 5, 80131 Naples, Italy.
}

Received: 26 September 2016 Accepted: 29 September 2016 Published online: 05 December 2016

\section{Reference}

1. Destefanis S, Giretto D, Muscolo MC, Di Cerbo A, Guidetti G, Canello S, Giovazzino A, Centenaro S, Terrazzano G. Clinical evaluation of a nutraceutical diet as an adjuvant to pharmacological treatment in dogs affected by Keratoconjunctivitis sicca. BMC Vet Res. 2016;12:214. doi:10.1186/s12917-016-0841-2.

\footnotetext{
* Correspondence: sarac@forza10.com

${ }^{\dagger}$ Equal contributors

${ }^{5}$ Research and Development Department, SANYpet S.p.a., Bagnoli di Sopra, Padua, Italy

Full list of author information is available at the end of the article
} 

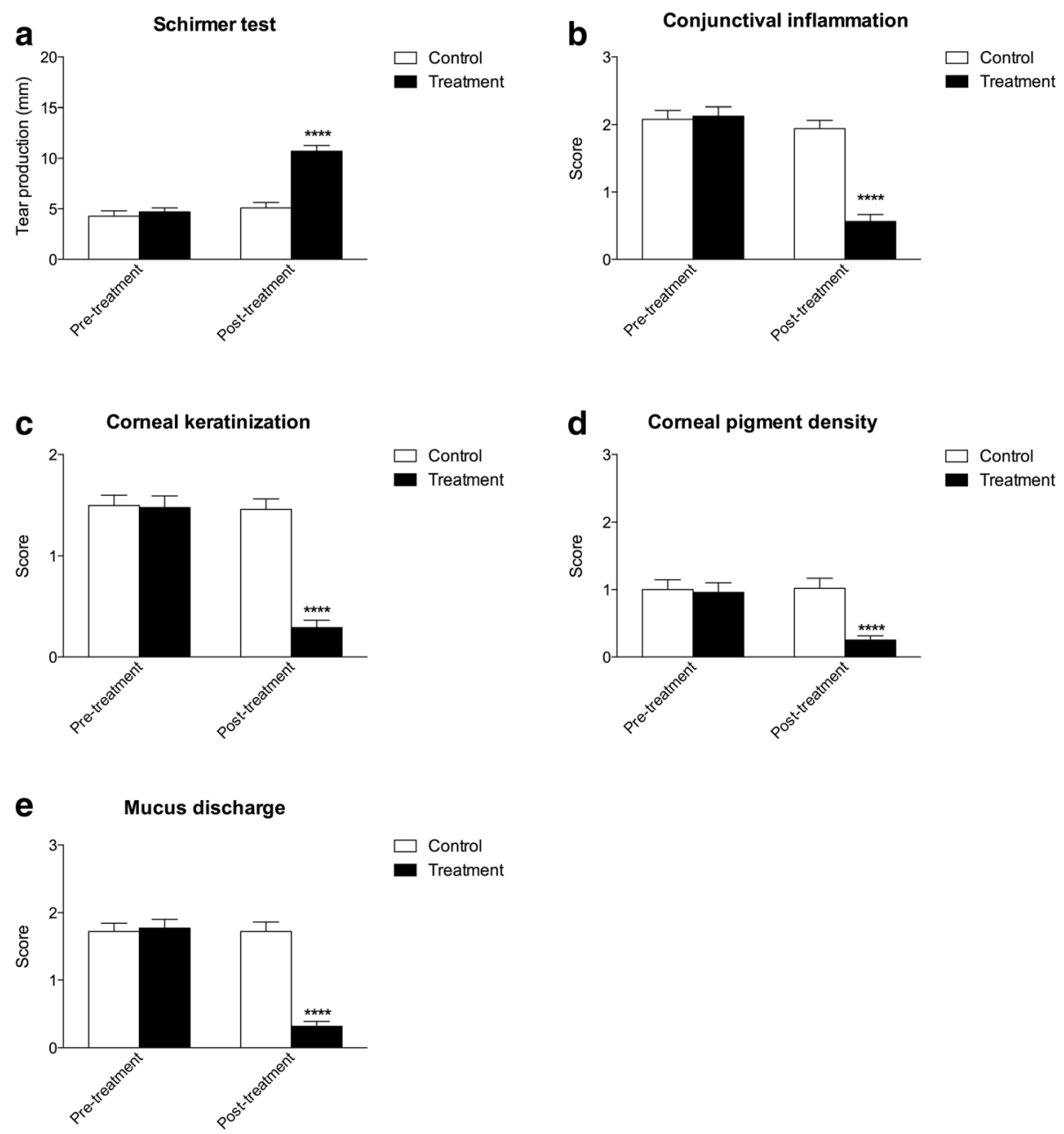

Fig. 2 a mean tear production (STT) in $\mathrm{mm} / \mathrm{min}$ before and after 60 days treatment for ND and SD group, STT values resulted significantly increased $\left({ }^{* * * *} P<0.0001\right)$ in ND group at the end of treatment, $\mathbf{b}$ Mean conjunctival inflammation scores before and after 60 days treatment for ND and SD group, a significant decrease $\left.{ }^{* * * *} P<0.0001\right)$ was observed in ND group at the end of the treatment; $\mathbf{c}$ mean corneal keratinization scores before and after 60 days treatment for ND and SD group, a significant decrease (***P $<0.0001$ ) was observed in ND group at the end of the treatment; $\mathbf{d}$ mean corneal pigment density scores before and after 60 days treatment for ND and SD group, a significant decrease (*** $P<0.0001)$ was observed in ND group at the end of the treatment; e mean mucus discharge scores before and after 60 days treatment for ND and SD group, a significant decrease $\left.{ }^{* * * *} P<0.0001\right)$ was observed in ND group at the end of the treatment 